\title{
Database Design on Experimental Equipment Accounting Treatment System based on Oracle
}

\author{
Yanmei Wang ${ }^{\mathrm{a}}$, Linchuang Zhang ${ }^{\mathrm{b}}$ \\ College of Information Science and Technology, Bohai University, Jinzhou, 121013, China \\ a19296571@qq.com, bzhanglinchuang123@163.com
}

\begin{abstract}
Keywords: experimental equipment; accounting treatment; database design; Oracle; conceptual structure; logical structure; integrity
\end{abstract}

\begin{abstract}
In order to test equipment purchase, use and inventory management in an all-round way, this paper based on needs analysis, in accordance with the standard design process research, provide technical support for system development. First of all, the conceptual structure design, using entity relationship diagram constructs the conceptual model; then, the logical structure design, with class diagram to express the logical structure based on Oracle; Finally, the design of integrity, includes entity integrity and referential integrity. In this paper, the design results are reasonable storage structure and access efficiency, according to the specific circumstances of the appropriate extension of the logical structure for the actual development, in order to adapt to the actual needs of system development.
\end{abstract}

\section{Introduction}

Experimental equipment is an important part of the experimental teaching security system, and timely, accurate, economical and efficient providing experimental equipment is the necessary condition to ensure the smooth implementation of the experiment. It has great significance for experimental equipment management in strengthening the inventory management of experimental equipment, reasonably determining the inventory of equipment and improving the use of experimental funds. The traditional manual management of university experimental equipment has many disadvantages, such as the not standardized system, cross-repeated configuration, low information transparency and inconsistent accounts. With the deepening of the reform of higher education, the standardization, scientific and information management of experimental equipment has become an important issue. Development of experimental equipment accounting system can all-round manage the purchase, requisition and inventory of experimental equipment, to keep abreast of the changes and the reasons in experimental equipment, so that unified accounts can be done to improve the use rate and information management level of experimental equipment [1-3]. Database design is an important work of software development, and this paper is based on the analysis and Oracle data management system to construct the optimal database model in accordance with the database design steps, so that it can effectively store data to meet a variety of user application requirements, which can provide technical support for software system development.

\section{Brief Requirements Analysis}

The core of experimental equipment accounting treatment system is the accounting treatment to manage all the information of experimental equipment. In software development, the use case is a main line throughout the system development, and the use case model is the system's established function (that is a model of system environment). Using the use case model can simplify and generalize the reality and enhance the understanding of the real building system. The users of this system mainly include purchasing managers, laboratory managers and account managers. As a complete information system, it includes many use cases, the core use cases are summarized as supplier management, equipment storage management, equipment category management, accounting treatment, equipment coding, equipment requisition management and laboratory management. Briefly described as follows according to the users: 
(1) The purchasing manager is responsible for the procurement of experimental equipment, so involving the system functions include: supplier management, that to manage the related information of supplier; equipment storage management, that entry information of equipment storage receipts; accounting treatment, that view equipment storage and inventory information.

(2) The laboratory manager is responsible for the requisition and management of experimental equipment, involving the system functions include: laboratory management, that manage laboratory-related information; equipment requisition management, that entry information of equipment requisition receipts; accounting treatment, that view equipment requisition and inventory information.

(3) The account manager is responsible for managing the accounts of the equipment, involving the system functions include: equipment category management, that classify equipment and maintain of classification information; equipment coding, that establish classification coding equipment to achieve standardized management; accounting treatment, that establish a detailed account page and maintain account page registration information.

\section{Conceptual Structure Design}

The conceptual structure is the common of various data models. It has nothing to do with the specific database management system. It is a real model of the real world, abstracting the user's needs into the information structure. The design results are described by the Entity-Relationship (E-R) [7]. The use case model described in Figure 1 is abstracted into seven entities, namely equipment category, equipment coding, equipment account page, equipment account page details, supplier, equipment storage receipt, laboratory and equipment requisition receipt. There are eight "one-to-many" links and one "one-to-one" link between entities. An equipment corresponds to a "equipment coding", "equipment coding" and "equipment account page" is link of "one to one", that an equipment only exists an account page, a account page only corresponds to an equipment. Every time each of the storage or equipment used should be stored a record in the "equipment account page details", "equipment storage receipt" and "equipment account page details" is link of "one to many", that an equipment may exist multiple storage records for multiple types of equipment; but one storage record in equipment account page details corresponds to a record of storage receipt. The other "one-to-many" links are no longer described. The result of conceptual structure design is shown in Fig. 1. The entity attributes are omitted from the figure since each entity contains too many attributes.

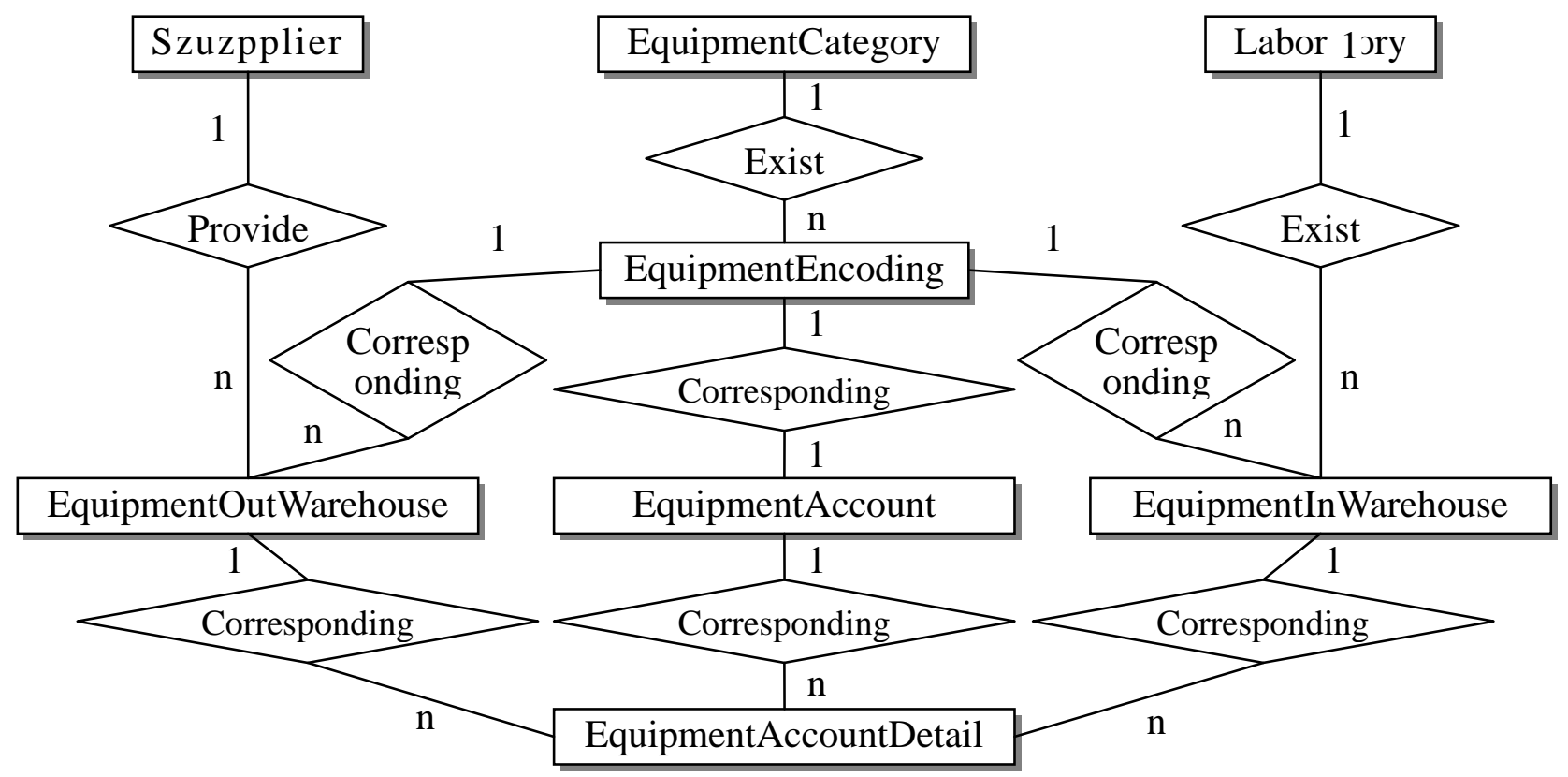

Fig.1. Entity-relationship diagram 


\section{Logical Structure Design}

The logical structure design is the process of converting the conceptual structure design result into the logical structure supported by the specific database management system. According to the conversion rule: an entity is converted into a relational mode; a "one-to-one" link can be converted into a separate relational mode, or it can be combined with any one of the corresponding relational modes; a "one-to-many" link can be converted to a separate relational mode, or it can also be combined with multiparty corresponding relational modes. Because there is no attribute of the connection in Figure 2, the conversion process only need to add the main key of another relationship mode to this the attribute of relational mode. The system based on Oracle database management system for logical structure design, Oracle contains a variety of data type, in order to facilitate the realization of the program, and the system selects Char, Varchar2, Number, Clob and Date types. Result of logic design uses class diagram to describe, as shown in Figure 2 [8-11]. As a complete management information system, due to management information to much and limited of the length of the article and the size of the restrictions, the attributes of "supplier", "equipment storage receipt", "laboratory" and "equipment requisition receipt" are omitted.

\section{Integrity Design}

The usual database integrity includes entity integrity, referential integrity, and user-defined integrity [12]. User-defined integrity is the setting of the range and value rules of the field in the database implementation phase. This paper only carries on the other two integrity design.

(1) Entity Integrity. Entity integrity refers to the integrity of records in the table, through the primary key field to achieve. The primary key can be consisted of a single field, or a combination of multiple fields, which requiring unique and not empty. The primary key name conforms to the naming rule of the database management system. The primary key name of this article consists of "PK _ + "table name". For the "EquipmentCategory" table, the primary key field is single field of "CategoryCode", the primary key name is "PK_EquipmentCategory"; for the "EquipmentInWarehouseInformation" table, the primary key field is union field of "EquipmentCode + InputBillNo", the primary key name is "PK_EquipmentInWarehouseInformation". The primary key fields of the other tables are marked in bold in Fig. 2.

(2) Referential Integrity. Referential integrity is guaranteed to ensure consistency between the data in the guaranteed table, through the foreign key to achieve, the purpose of it is to modify, delete, insert a data in table, we can reference to use related data in another table to check the operation is correct or not, which requiring the value must be a valid value of the primary table and primary key from foreign key or an "empty" value [13]. For the "EquipmentCategory" table and the "EquipmentEncoding" table, the "CategoryCode" field is the primary key of the "EquipmentCategory" table and the foreign key of the "EquipmentEncoding" table, so the foreign key "FK_CategoryCode" created according to the "CategoryCode" field can ensure the referential integrity between two tables. Referential integrity design results of other tables are marked in Fig. 2, the lines with arrows show the relationship between the two tables, the arrow pointing to is the main table, the other is the slave table, the field with underlined is foreign key, "FK_" at the beginning next to arrow line is the foreign key name.

\section{Conclusion}

Experimental equipment management is an important work of laboratory management in university. The accounting treatment system solves the core problem of experimental equipment management, which is of great significance to reduce inventory cost, improve equipment efficiency and improve fund utilization rate. This paper is designed according to the standardized method, which has the characteristics of reasonable storage structure and high access efficiency, and it can meet the needs of different personnel. In specific application, it can be expand appropriately for the logical 
structure according to the actual situation to meet the actual needs of program development and data management.

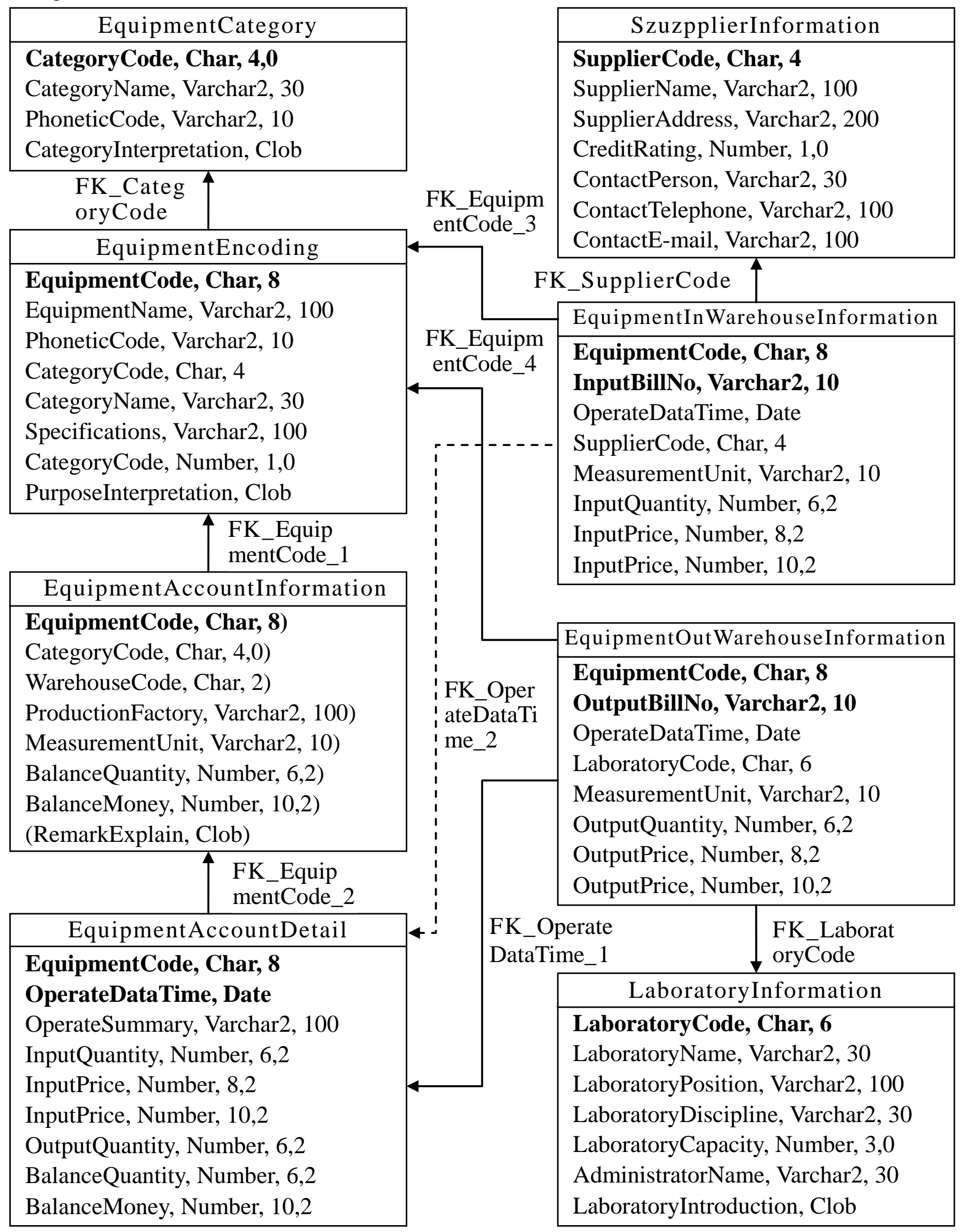

Fig.2. Database logical structure model of experimental equipment accounting treatment system 


\section{References}

[1] W. F. Ji, G. Y. Wang, H. F. Xu, X. Q. Zhang, "Inventory control of laboratory equipment based on Supply Chain Management," Laboratory research and exploration, vol. 34, no. 1, pp. 274-276, 2015.

[2] Q. L. Huang, W. F. Ji, M. Zhao, "Research on the application of experiment equipment support based on neural network technology," Experimental technology and management, vol. 31, no. 7, pp. 235-236, 2014.

[3] N. Wei, "Strengthen the management of equipment, improve the efficiency of experimental teaching," Chinese modern educational equipment, vol. 17, no. 14, pp. 7-8, 2014.

[4] Y. Wang, Q. Meng, X. Liu, R. Wang, "Research on the application of RFID in inventory management of laboratory equipment," Experimental technology and management, vol. 32, no. 11, pp. 276-279, 2015.

[5] X. X. Lv, "Design of database management system for music equipment management," Electronic test, vol. 21, no. 14, pp. 37-38, 2014.

[6] V.V. Afanasyev, E.F. Kryuchkov, V.I. Petrov, et al., "Virtual Labs on Unique Experimental Equipment," Procedia - Social and Behavioral Sciences, vol. 128, no. 22, pp. 482-488, 2014.

[7] X. Teng. Database Design of Physics Experimental Equipment Management System[J]. AER-Advances in Engineering Research, 2005, 15(1): 398-401.

[8] H Y. Liu, W. B. Hu, "Development of experimental equipment management system with .NET2.0," Experimental science and technology, vol. 5, no. 5, pp. 75-77, 2007.

[9] Vladimir Dimitrieski, Milan Čeliković, "Concepts and evaluation of the extended entity-relationship approach to database design in a multi-paradigm information system modeling tool," Computer Languages, Systems \& Structures, vol. 44, no. 3, pp. 299-318, 2015.

[10] L. Shen, "The importance of data integrity in the course of network database and its teaching," Journal of Mudanjiang Institute of Education, vol. 32, no. 9, pp. 104-105, 2014.

[11] C. H. Zhai, W. G. Zhang, C. H. Zhang, "Construction practice of integrated management information system for laboratory equipment," Experimental technology and management, vol. 32, no. 5, pp. 153-156, 2015.

[12] Y. S. Huang, Y. H. Cao, "Dynamic design and implementation of database referential integrity," Electronic production, vol. 32, no. 11, pp. 76-77, 2015.

[13] X. X. LV, "The design of data integrity in the accounting management system of music equipment management," Electronic test, vol.21, no. 16, pp. 11-12, 2014. 